\title{
ON MATROIDS AND
}

\section{LINEARLY INDEPENDENT SET FAMILIES}

\author{
Giuliano G. La Guardia ${ }^{1 \S}$, Luciane Grossi ${ }^{2}$, Welington Santos ${ }^{3}$ \\ ${ }^{1,2}$ Department of Mathematics and Statistics \\ State University of Ponta Grossa \\ 84030-900, Ponta Grossa - PR, BRAZIL \\ ${ }^{3}$ Department of Mathematics \\ Federal University of Parana, \\ CP 19.011, 81.531-990, Curitiba, PR, BRAZIL
}

\begin{abstract}
New families of matroids are constructed in this note. These new families are derived from the concept of linearly independent set family (LISF) introduced by Eicker and Ewald [Linear Algebra and its Applications 388 (2004) 173-191]. The proposed construction generalizes in a natural way the well known class of vectorial matroids over a field.
\end{abstract}

AMS Subject Classification: 81Q99

Key Words: matroid theory, linearly independent set family

\section{Introduction}

In his seminal paper [7] on matroid theory, Hassler Whitney dealt with the problem of characterizing matroids that are representable over a given field (see also the interesting papers [1, 2, 4]). In fact, as it is well known, the matroid theory is a powerful tool in order to study several classes endowed with algebraic structures such as, affine spaces, vector spaces, algebraic independence, graph theory and so on. Among these classes, a particular class is of essential importance: the class of vectorial matroids.

Received: May 4, 2015

Published: February 9, 2016

$\S$ Correspondence author
(C) 2016 Academic Publications, Ltd. url: www.acadpubl.eu 
In this note we generalize the class of vectorial matroid by applying the concept of linearly independent set family (LISF), introduced by Eicker and Ewald [3], which extends in a straightforward way the definition of linearly independent vectors to independent sets in a vector space. More precisely, the LISF's have essential ingredients in order to provide a natural generalization of the class of vectorial matroids over a given field.

Section 2 presents basic concepts on matroid theory and linearly independent set family, necessary for the development of this note. In Section 3, we present the contributions of this paper: a new class of matroids derived from linearly independent set families are constructed. In Section 4, the final remarks are drawn.

\section{Preliminaries}

This section is concerned with a review of matroid theory $[6,5]$ as well as the review of the concept of linearly independent set family (LISF) [3].

\subsection{Matroid Theory}

As was said previously we utilize the definition of matroid based on independent sets (although the other definitions are equivalents). The following basic concepts can be found in [5].

Definition 1. A matroid $M$ is an ordered pair $(S, \mathcal{I})$ consisting of a finite set $S$ and a collection $\mathcal{I}$ of subsets of $S$ satisfying the following three conditions:

(I.1) $\emptyset \in \mathcal{I}$;

(I.2) If $I \in \mathcal{I}$ and $I^{\prime} \subset I$, then $I^{\prime} \in \mathcal{I}$;

(I.3) If $I_{1}, I_{2} \in \mathcal{I}$ and $\left|I_{1}\right|<\left|I_{2}\right|$, then there exists an element $e \in I_{2}-I_{1}$ such that $I_{1} \cup\{e\} \in \mathcal{I}$, where $\mid$. $\mid$ denote the cardinality of the set.

If $M$ is the matroid $(S, \mathcal{I})$, then $M$ is called matroid on $S$. The members of $\mathcal{I}$ are independent sets of $M$, and $S$ is the ground set of $M$. A subset of $S$ that is not in $\mathcal{I}$ is called dependent. Minimal dependent sets are dependents sets all of whose proper subsets are independents. Minimal dependent sets are called circuits of $M$. An independent set is called maximal if the inclusion of any element in this set results in a dependent set. Maximal independent sets are called basis of the matroid. It is well known that a matroid can be defined in many different (but equivalent) ways, i. e., by means of independent sets, circuits, basis and so on. In our case we consider the definition of matroid based on independent sets, as given above. 
Let us recall the well known concept of vectorial matroid:

Theorem 2. Let $S$ be the set of column labels of a matrix $A_{m \times n}$ over a field $\mathbb{F}$, and let $\mathcal{I}$ be the set of subsets $X$ of $S$ for which the multiset of columns labelled by $X$ is linearly independent $(L I)$ in $V(m, F)$, the $m$-dimensional vector space over $\mathbb{F}$. Then $(S, \mathcal{I})$ is a matroid.

\subsection{Linearly Independent set Families}

The concept of linearly independent set families was introduced by Eicker and Ewald in [3]. This definition extends in a straightforward way the definition of linearly independent vectors to independent sets in a vector space. Although our definition is different from the original one, it contains essentially the same idea contained in [3].

Definition 3. Let $\mathbb{V}$ be a $l$-dimensional vector space over a field $\mathbb{F}$. A family $\mathcal{J}$ of non-empty subsets $C_{i} \subset \mathbb{V}$, where $i=1, \ldots, n(n \leq l)$, given by $\mathcal{J}:=\left\{C_{i}, i=1, \ldots, n\right\}$ is called a linearly independent set family (LISF) if and only if any selection of $n$ vectors $v_{i} \in C_{i}$ is linearly independent in $\mathbb{V}$.

Example 4. The first and trivial example of LISF is a set of $n \leq l$ linearly independent vectors in $\mathbb{R}^{l}$. As a second illustrative example, consider in $\mathbb{R}^{2}$ the open quadrants $C_{1}:=\left\{\left(x_{1}, x_{2}\right): x_{1}>0, x_{2}>0\right\}$, and $C_{2}:=\left\{\left(x_{1}, x_{2}\right): x_{1}>\right.$ $\left.0, x_{2}<0\right\}$. Then $\mathcal{F}:=\left\{C_{1}, C_{2}\right\}$ is a LISF.

\section{The Results}

In this section we present the contributions of the paper. Theorems 5 and 11 generalize the well known class of vectorial matroids over a given field, consequently, new families of matroids are obtained.

Theorem 5. Consider that $n \geq 1$ and $l \geq 1$ are integers. Let $E_{1}, E_{2}, \ldots, E_{n}$ be subsets of a finite dimensional vector space $\mathbb{V}$ over a field $\mathbb{F}$ such that $E_{i} \subset \mathbb{W}_{i}$ for all $i=1, \ldots, n$, where $\mathbb{W}_{i}$ are one-dimensional subspaces of $\mathbb{V}$. Consider the multiset of labels $S=\{1, \ldots, n\}$, and let $\mathcal{I}$ be the set of subsets $I=\left\{i_{1}, \ldots, i_{j}\right\}$ of $S$ for which $\left\{E_{i_{1}}, E_{i_{2}}, \ldots, E_{i_{j}}\right\}$ form a $L I S F$. Then the ordered pair $(S, \mathcal{I})$ is a matroid.

Proof. We must prove that $(S, \mathcal{I})$ satisfies (I.1), (I.2) and (I.3) of Definition 1. Properties (I.1) and (I.2) are clearly satisfied. 
Let us now show that (I.3) holds. Seeking a contradiction, suppose that (I.3) does not hold. Consider that $I_{1}, I_{2} \in \mathcal{I}$ with $\left|I_{1}\right|<\left|I_{2}\right|$, where $I_{1}=\left\{i_{a_{1}}, \ldots, i_{a_{j}}\right\}$ and $I_{2}=\left\{i_{b_{1}}, \ldots, i_{b_{k}}\right\}, j<k$. Then for each $e \in I_{2}-I_{1}$ it follows that $I_{1} \cup\{e\} \notin \mathcal{I}$. We know that $\left\{E_{i_{a_{1}}}, E_{i_{a_{2}}}, \ldots, E_{i_{a_{j}}}\right\}$ and $\left\{E_{i_{b_{1}}}, E_{i_{b_{2}}}, \ldots, E_{i_{b_{k}}}\right\}$ are LISF's. Since $I_{1} \cup\{e\} \notin \mathcal{I}$ for each $e \in I_{2}-I_{1}$, then the sets $\left\{E_{i_{a_{1}}}, E_{i_{a_{2}}}, \ldots\right.$, $\left.E_{i_{a_{j}}}, E_{e}\right\}$ does not form a LISF for each $e \in I_{2}-I_{1}$. Fix $e \in I_{2}-I_{1}$. Then there exist vectors $\mathbf{v}_{l} \in E_{i_{a_{l}}}$, where $1 \leq l \leq j$, and $\mathbf{x} \in E_{e}$ such that $\mathbf{v}_{1}, \ldots, \mathbf{v}_{j}, \mathbf{x}$ are linearly dependents in $\mathbb{V}$. Hence there exist $\alpha_{x}, \alpha_{l} \in \mathbb{F}, 1 \leq l \leq j$, with $\alpha_{x} \neq 0$ such that $\alpha_{1} \mathbf{v}_{1}+\ldots+\alpha_{j} \mathbf{v}_{j}+\alpha_{x} \mathbf{x}=0$, otherwise the unique solution for the last equality would be $\alpha_{x}=\alpha_{l}=0$ for each $1 \leq l \leq j$, which is a contradiction. This means that $\mathbf{x}=\left(-\alpha_{1} \alpha_{x}^{-1}\right) \mathbf{v}_{1}+\ldots+\left(-\alpha_{j} \alpha_{x}^{-1}\right) \mathbf{v}_{j}$. For every vector $\mathbf{w} \in E_{e}$ we have $\mathbf{w}=\beta \mathbf{x}=\left(-\alpha_{1} \beta \alpha_{x}^{-1}\right) \mathbf{v}_{1}+\ldots+\left(-\alpha_{j} \beta \alpha_{x}^{-1}\right) \mathbf{v}_{j}, \beta \in \mathbb{F}$, because $E_{e} \subset \mathbb{W}_{e}$ and $\mathbb{W}_{e}$ is an one-dimensional subspace of $\mathbb{V}$. Thus the subspace $\mathbb{U}_{e}$ spanned by the sets $E_{i_{a_{1}}}, E_{i_{a_{2}}}, \ldots, E_{i_{a_{j}}}, E_{e}$, is contained in the subspace $\mathbb{Y}$ spanned by $E_{i_{a_{1}}}, E_{i_{a_{2}}}, \ldots, E_{i_{a_{j}}}$, for each $e \in I_{2}-I_{1}$. Consequently, the subspace $\mathbb{X}$ spanned by $E_{i_{a_{1}}}, E_{i_{a_{2}}}, \ldots, E_{i_{a_{j}}}, E_{i_{b_{1}}}, E_{i_{b_{2}}}, \ldots, E_{i_{b_{k}}}$, is also contained in $\mathbb{Y}$, so it follows that $\left|I_{2}\right| \leq \operatorname{dim}(\mathbb{W}) \leq\left|I_{1}\right|<\left|I_{2}\right|$, which is a contradiction. Therefore the ordered pair $(S, \mathcal{I})$ is a matroid.

In the following corollaries of Theorem 5, one can generate more families of matroids:

Corollary 6. Suppose that $n \geq 1$ and $l \geq 1$ are integers. Let $E_{1}, E_{2}, \ldots, E_{n}$ be subsets of a finite dimensional vector space $\mathbb{V}$ such that $E_{i} \subset \mathbb{W}_{i}$ for all $i=1, \ldots, n$, where $\mathbb{W}_{i}$ are one-dimensional subspaces of $\mathbb{V}$. Consider the multiset of labels $S=\{1, \ldots, n\}$, and let $\mathcal{I}$ be the set of subsets $I=\left\{i_{1}, \ldots, i_{j}\right\}$ of $S$ for which $\left\{\lambda_{i_{1}} E_{i_{1}}, \lambda_{i_{2}} E_{i_{2}}, \ldots, \lambda_{i_{j}} E_{i_{j}}\right\}$ form a LISF, where $\lambda_{i_{r}} \neq 0$ for all $r=1, \ldots, j$. Then the ordered pair $(S, \mathcal{I})$ is a matroid.

Proof. It follows from the fact that $\left\{E_{i_{1}}, E_{i_{2}}, \ldots, E_{i_{j}}\right\}$ is a LISF if and only if $\left\{\lambda_{i_{1}} E_{i_{1}}, \lambda_{i_{2}} E_{i_{2}}, \ldots, \lambda_{i_{j}} E_{i_{j}}\right\}$ is a LISF, where $\lambda_{i_{r}} \neq 0$ for all $r=1, \ldots, j$.

Corollary 7. Consider that $n \geq 1$ and $l \geq 1$ are integers and let $E_{1}, E_{2}, \ldots, E_{n}$ be subsets of a finite dimensional vector space $\mathbb{V}$ such that $E_{i} \subset \mathbb{W}_{i}$ for all $i=1, \ldots, n$, where $\mathbb{W}_{i}$ are one-dimensional subspaces of $\mathbb{V}$. Assume that $S=\{1, \ldots, n\}$ is the multiset of labels and $\mathcal{I}$ is the set of subsets $I=\left\{i_{1}, \ldots, i_{j}\right\}$ of $S$ such that $\left\{T\left(E_{i_{1}}\right), T\left(E_{i_{2}}\right), \ldots, T\left(E_{i_{j}}\right)\right\}$ form a LISF, for any isomorphism $T$ on $\mathbb{V}$. Then the ordered pair $(S, \mathcal{I})$ is a matroid.

Proof. This is true due to the fact that $\left\{E_{i_{1}}, E_{i_{2}}, \ldots, E_{i_{j}}\right\}$ form a LISF if and only if $\left\{T\left(E_{i_{1}}\right), T\left(E_{i_{2}}\right), \ldots, T\left(E_{i_{j}}\right)\right\}$ form a LISF. 
Corollary 8. Consider that $n \geq 1$ and $l \geq 1$ are integers. Let $E_{1}, E_{2}, \ldots, E_{n}$ be subsets of a finite dimensional vector space $\mathbb{V}$ such that $E_{i} \subset \mathbb{W}_{i}$ for all $i=1, \ldots, n$, where $\mathbb{W}_{i}$ are one-dimensional subspaces of $\mathbb{V}$. Consider the multiset of labels $S=\{1, \ldots, n\}$ and let $\mathcal{I}$ be the set of subsets $I=\left\{i_{1}, \ldots, i_{j}\right\}$ of $S$ such that $\left\{E_{i_{1}} \cup\left(-E_{i_{1}}\right), E_{i_{2}} \cup\left(-E_{i_{2}}\right), \ldots, E_{i_{j}} \cup\left(-E_{i_{j}}\right)\right\}$ form a LISF. Then the ordered pair $(S, \mathcal{I})$ is a matroid.

Proof. Follows from the fact that $\left\{E_{i_{1}}, E_{i_{2}}, \ldots, E_{i_{j}}\right\}$ is a LISF if and only if $\left\{E_{i_{1}} \cup\left(-E_{i_{1}}\right), E_{i_{2}} \cup\left(-E_{i_{2}}\right), \ldots, E_{i_{j}} \cup\left(-E_{i_{j}}\right)\right\}$ is a LISF.

In the following examples we presents two LISF's that does not form a matroid:

Example 9. Consider the (real) vector space $\mathbb{R}^{2}$ and the following subsets $E_{1}, E_{2}, E_{3}$ of $\mathbb{R}^{2}$ given by: $E_{1}=\left\{(x, y) \in \mathbb{R}^{2} \mid(x-1)^{2}+(y-1)^{2} \leq 1\right\} ; E_{2}=$ $\left\{(x, y) \in \mathbb{R}^{2} \mid(x-1)^{2}+y^{2} \leq 1\right\}\{(0,0)\} ; E_{3}=\left\{(x, y) \in \mathbb{R}^{2} \mid(x-1)^{2}+(y+1)^{2} \leq\right.$ $1 / 9\}$. Assume that $S=\{1,2,3\}$ and consider that $I=\left\{i_{1}, \ldots, i_{j}\right\} \in \mathcal{I}$ if and only if the sets $\left\{E_{i_{1}}, E_{i_{2}}, \ldots, E_{i_{j}}\right\}$ form a LISF. From construction and applying the same notation as in Theorem 5, one has $\mathcal{I}=\{\emptyset,\{1\},\{2\},,\{3\},\{1,3\}\}$. But since $|\{2\}|<|\{1,3\}|$, one concludes from (I.3) that $\{1,2\} \in \mathcal{I}$ or $\{2,3\} \in \mathcal{I}$, a contradiction.

Example 10. Consider now the (real) vector space $\mathbb{R}^{3}$ and the following subsets $E_{1}, E_{2}, E_{3}$ of $\mathbb{R}^{3}$ given by: $E_{1}=\left\{(x, y, z) \in \mathbb{R}^{3} \mid(x, y, z)=(0,0,0)+\right.$ $a(1,0,0)+b(0,1,0) ; a, b \in \mathbb{R}\}-\{(0, y, 0) \mid y \in \mathbb{R}\} ; E_{2}=\left\{(x, y, z) \in \mathbb{R}^{3} \mid(x, y, z)=\right.$ $(0,0,0)+c(0,0,1)+d(1,1,0) ; c, d \in \mathbb{R}\}-\left\{(0,0,0\} ; E_{3}=\left\{(x, y, z) \in \mathbb{R}^{3} \mid(x, y, z)=\right.\right.$ $(0,0,0)+e(0,1,0)+f(0,0,1) ; e, f \in \mathbb{R}\}-\{(0, y, 0) \mid y \in \mathbb{R}\}$. As in the previous example, if $S=\{1,2,3\}$, from construction we have $\mathcal{I}=\{\emptyset,\{1\},\{2\},,\{3\}$, $\{1,3\}\}$. However, since $|\{2\}|<|\{1,3\}|$, it follows from (I.3) that $\{1,2\} \in \mathcal{I}$ or $\{2,3\} \in \mathcal{I}$, a contradiction.

However, under suitable hypothesis one can get the following:

Theorem 11. Let $\mathbb{F}$ be a field of characteristic zero. Assume that $\mathbb{V}=$ $\mathbb{W}_{1} \oplus \ldots \oplus \mathbb{W}_{k}$ is a vector space over $\mathbb{F}$ that is the direct sum of $n$-dimensional subspaces $\mathbb{W}_{i}, i=1, \ldots, k$. Let $E_{1}, \ldots, E_{m}$ be subsets of $\mathbb{V}$ such that for each $i=1, \ldots, m, E_{i}$ contains (with exception of the zero vector) an $n_{E_{i}}$ dimensional subspace of $\mathbb{V}$, where $\lceil n / 2\rceil+1 \leq n_{E_{i}} \leq n$, and $E_{i} \subset \mathbb{W}_{i^{*}}$ for some $1 \leq i^{*} \leq k$. If $S=\{1, \ldots, m\}$ is the multiset of labels and $\mathcal{I}$ is the set of subsets $I=\left\{i_{1}, \ldots, i_{r}\right\}$ of $S$ such that $\left\{E_{i_{1}}, E_{i_{2}}, \ldots, E_{i_{r}}\right\}$ form a LISF, then the ordered pair $(S, \mathcal{I})$ is a matroid. 
Proof. Obviously (I.1) and (I.2) are satisfied. We will prove (I.3). For, assume that $I_{1}, I_{2} \in \mathcal{I}$ with $\left|I_{1}\right|<\left|I_{2}\right|$ and $I_{1}=\left\{i_{1}, \ldots, i_{s}\right\}$ and $I_{2}=\left\{j_{1}, \ldots, j_{t}\right\}$, where $s<t$. Thus the sets $\left\{E_{i_{1}}, E_{i_{2}}, \ldots, E_{i_{s}}\right\}$ form a LISF and, from hypothesis, each of these sets is contained in distinct $\mathbb{W}_{i}$ 's. These facts also hold for the sets $\left\{E_{j_{1}}, E_{j_{2}}, \ldots, E_{j_{t}}\right\}$ corresponding to $I_{2}$. Suppose without loss of generality (w.l.g.) that $E_{i_{1}} \subset \mathbb{W}_{1}, \ldots, E_{i_{s}} \subset \mathbb{W}_{s}$. Since $\left|I_{1}\right|<\left|I_{2}\right|$ then there exists an $\mathbb{W}_{s^{*}} \neq \mathbb{W}_{1}, \mathbb{W}_{2}, \ldots, \mathbb{W}_{s}$ such that $E_{j_{s+1}} \subset \mathbb{W}_{s^{*}}$. This is possible due to the fact that each of the sets $E_{j_{1}}, E_{j_{2}}, \ldots, E_{j_{t}}$ is contained in distinct $\mathbb{W}_{i}$ 's and $s<t$. Consider the sets $E_{i_{1}}, E_{i_{2}}, \ldots, E_{i_{s}}, E_{j_{s+1}}$. For every choice of vectors $\mathbf{v}_{i_{1}} \in E_{i_{1}}, \ldots, \mathbf{v}_{i_{s}} \in E_{i_{s}}$ and $\mathbf{v}_{j_{s+1}} \in E_{j_{s+1}}$, we claim that the vectors $\mathbf{v}_{i_{1}}, \ldots, \mathbf{v}_{i_{s}}, \mathbf{v}_{j_{s+1}}$ are linearly independents. In fact, seeking a contradiction we assume that the vectors are linearly dependents. W.l.g., suppose that $a_{i_{1}} \mathbf{v}_{i_{1}}+\ldots+a_{i_{s}} \mathbf{v}_{i_{s}}+b_{j_{s+1}} \mathbf{v}_{j_{s+1}}=0$, where $a_{i_{1}}, \ldots, a_{i_{s}}, b_{j_{s+1}} \in \mathbb{F}$, and $b_{j_{s+1}} \neq 0$ then $\left(a_{i_{1}} b_{j_{s+1}}^{-1}\right) \mathbf{v}_{i_{1}}+\ldots+\left(a_{i_{s}} b_{j_{s+1}}^{-1}\right) \mathbf{v}_{i_{s}}+\mathbf{v}_{j_{s+1}}=0$. Since $\left(a_{i_{1}} b_{j_{s+1}}^{-1}\right) \mathbf{v}_{i_{1}} \in$ $\mathbb{W}_{1}, \ldots,\left(a_{i_{s}} b_{j_{s+1}}^{-1}\right) \mathbf{v}_{i_{s}} \in \mathbb{W}_{s}$ and $\mathbf{v}_{j_{s+1}} \in \mathbb{W}_{s^{*}}$, then one has $\mathbf{v}_{j_{s+1}}=0$, which is a contradiction. The cases for which $a_{i_{1}} \mathbf{v}_{i_{1}}+\ldots+a_{i_{s}} \mathbf{v}_{i_{s}}+b_{j_{s+1}} \mathbf{v}_{j_{s+1}}=0$, where $a_{i_{1}}, a_{i_{2}}, \ldots, a_{i_{s}}, b_{j_{s+1}} \in \mathbb{F}$, and $a_{i_{l}} \neq 0$ for some $l=1, \ldots, s$ are analogous. Thus the sets $\left\{E_{i_{1}}, E_{i_{2}}, \ldots, E_{i_{s}}, E_{j_{s+1}}\right\}$ form a LISF, so $I_{1} \cup\left\{j_{s+1}\right\} \in \mathcal{I}$, where $j_{s+1} \in I_{2}-I_{1}$. Therefore, the ordered pair $(S, \mathcal{I})$ is a matroid and the proof is complete.

\section{Summary}

We have constructed new families of matroids derived from linearly independent set families. The presented construction generalizes in a natural way the class of vectorial matroids over a field.

\section{Acknowledgment}

This research was partially supported by the Brazilian Agencies CAPES and CNPq.

\section{References}

[1] R. A. Brualdi, Comments on bases in dependence structures, Bull. Australian Math. Society, 1 (1969), 161-167. 
[2] T. H. Brylawski, Some Properties of Basic Families of Subsets, Discrete Math., 6 (1973), 333-341.

[3] F. Eicker and G. Ewald, Linearly independent set families, Linear Algebra and its Applications, 388 (2004), 173-191. 2004.

[4] C. Greene, Weight enumeration and the geometry of linear codes, Stud. Appl. Math., 55 (1976), 119-128.

[5] J. G. Oxley, Matroid Theory, Oxford University Press, New York, (1992).

[6] D. J. A. Welsh, Matroid Theory, Academic Press Inc., London L.T.D., (1976).

[7] H. Whitney, On the abstract properties of linear dependence, Amer. J. Math., 57 (1935), 509-533. 
